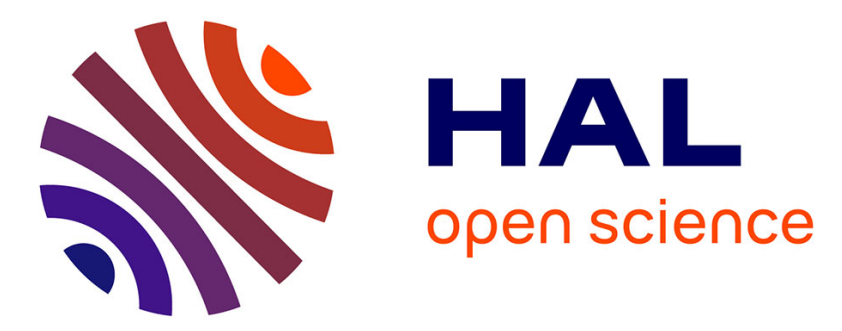

\title{
Parameter identifiability of ductile fracture criterion for DP steels using bi-level reduced surrogate model
}

\author{
Yuliang Hou, Qiaoli Zhao, Thaneshan Sapanathan, Alexandre Dumon, \\ Mohamed Rachik
}

\section{- To cite this version:}

Yuliang Hou, Qiaoli Zhao, Thaneshan Sapanathan, Alexandre Dumon, Mohamed Rachik. Parameter identifiability of ductile fracture criterion for DP steels using bi-level reduced surrogate model. Engineering Failure Analysis, 2019, 100, pp.300 - 311. 10.1016/j.engfailanal.2019.02.057 . hal-03485897

\section{HAL Id: hal-03485897 https://hal.science/hal-03485897}

Submitted on 20 Dec 2021

HAL is a multi-disciplinary open access archive for the deposit and dissemination of scientific research documents, whether they are published or not. The documents may come from teaching and research institutions in France or abroad, or from public or private research centers.
L'archive ouverte pluridisciplinaire HAL, est destinée au dépôt et à la diffusion de documents scientifiques de niveau recherche, publiés ou non, émanant des établissements d'enseignement et de recherche français ou étrangers, des laboratoires publics ou privés.

\section{(ㄷ)(1) $\$$}

Distributed under a Creative Commons Attribution - NonCommercial| 4.0 International 


\title{
Parameter identifiability of ductile fracture criterion for DP steels using bi-level reduced surrogate model
}

\author{
Yuliang Hou ${ }^{\mathrm{a}, \mathrm{b}, *}$, Qiaoli Zhao ${ }^{\mathrm{a}}$, Thaneshan Sapanathan ${ }^{\mathrm{b}, \mathrm{c}}$, Alexandre Dumon ${ }^{\mathrm{d}}$, Mohamed Rachik ${ }^{\mathrm{b}}$ \\ ${ }^{a}$ School of Mechanical Engineering, Zhengzhou University, Science Road 100, 450001, Zhengzhou, China \\ ${ }^{b}$ Laboratoire Roberval, CNRS UMR7337, Sorbonne université, Université de Technologie de Compiègne, Centre de \\ recherche Royallieu, CS60319, 60203, Compiègne Cedex, France \\ ${ }^{c}$ Institute of Mechanics, Materials and Civil Engineering, UCLouvain, 1348 Louvain-la-Neuve, Belgium \\ ${ }^{d}$ ESI Group, 99 Rue des Solets, BP80112, 94513, Rungis Cedex, France
}

\begin{abstract}
Generally, a large number of fracture tests are required to characterize the material parameters of the modified Mohr-Coulomb (MMC) fracture criterion. It takes enormous amount of time and expenses to prepare specimens and analyze experimental measurements. However, manufacturing industries are keen to seek fast and cost effective approaches. Therefore, a bi-level reduced surrogate model has been proposed as an alternative method for the parameter identification. Using this approach, the identification process becomes feasible and it requires only a limited number of experimental tests. The surrogate model is used in the current framework to empirically capture the non-linear evolution of material parameters for the fracture onset under the uniaxial loading condition. It assembles local critical elements associated with the global 3D finite element (FE) models. The surrogate model of fracture strain is constructed using Diffuse Approximation and the local elements to identify the unknown parameters. Then, global fracture simulations which consider the identified parameters, are preformed to update the target fracture strain and to compute the corresponding failure onset displacement. Satisfactory results are obtained by adopting the concept of successive Design of Experiments (DOE). The identification protocol is validated, and it is capable of calibrating ductile fracture parameters for different DP steels. Finally, a parametric study is performed to analyze the sensitivity of each free parameter and the influence of the used polynomial basis within the identification approach.
\end{abstract}

Keywords: Surrogate model, Diffuse Approximation, Parameter identification, Ductile fracture, Successive DOE

\section{Introduction}

Dual phase (DP) steels have a specific microstructure consisting of hard martensite islands imbedded in a soft and ductile ferrite matrix. DP steels have been increasingly used in various

\footnotetext{
*Corresponding author.

Email address: yulianghou@zzu.edu.cn (Yuliang Hou)
} 
structures and components which are included in modern carbody manufacturing due to their favorable combination of good strength and ductility [1-7]. However, formability of the DP steels are limited due to plastic localisation or fracture, in particular new generations of DP steels highly suffer from limited resistance to cracking (due to poor uniform elongation). For instance, cutting operations generate starter cracks at the edges of the sheets leading to limited open hole expansion. Thus, ductile fracture behaviors of DP steels become complex and it needs to be investigated and subsequently controlled during manufacturing processes [8]. To avoid the presence of ductile fracture in DP steels, reliable constitutive models and material parameters are required to predict the reliable fracture behaviors [6,9-15].

Recently, phenomenological models [16-20] have been developed to predict the ductile fracture behaviors without considering the nucleation, growth and coalescence of micro-voids. Development of these models was based on a reasonable assumption that the ductile fracture occurs when a weighting indicator of the accumulated plastic strain reaches a critical value. A comparative study of various phenomenological models with different types of weighting functions, was performed by Bao and Wierzbicki [9], using a series of experimental tests on 2024-T351 aluminum alloy. They reported that, all these models failed to effectively predict the ductile fracture for a certain material under various stress triaxialities. Hence, they concluded that, different weighting functions were required to obtain the accurate prediction of ductile fracture for a wide range of stress triaxialities, but no particular function type was provided. Based on the transformation formulas between the principal stresses and the stress state parameters (mainly stress triaxiality and Lode angle parameter), Bai and Wierzbicki $[16,17]$ extended the classical Mohr-Coulomb criterion into a mixed stress-strain fracture criterion to predict the onset of ductile fracture. It is also called as the modified Mohr-Coulomb (MMC) criterion, and the weighting indicator includes the equivalent plastic strain, stress triaxiality and Lode angle parameter. Although the MMC criterion has been well exploited with several metals, a careful executed calibration procedure is necessary for each material [16].

To identify the material parameters of these phenomenological models, many researchers have performed numerous experimental investigations for various ductile materials $[9,18]$. Dunand and Mohr [18] carried out a large number of fracture experiments on TRIP780 steel to identify the parameters of the MMC fracture criterion. Thus, it costs both time and expenses to prepare specimens and to perform the experimental measurements. It is the main drawback when identifying material parameter for a DP steel in practical engineering applications. Therefore, researchers in $R \& D$ and academic are keen to seek a fast and low-cost approach to overcome this challenge. Hence, a bi-level reduced surrogate model has been developed as a computationally inexpensive alternative to identify material parameters of MMC fracture criterion [6]. In the method, it assembles local critical element models associated with global 3D finite element (FE) models as the source of training data to identify the material parameters of MMC criterion for a given DP steel. Then, for a given fracture onset displacements and fracture strains, the bi-level reduced surrogate model is constructed using Diffuse Approximation [21, 22] and successive Design of Experiments (DOE). However, limited discussions on the reliability of this identification approach was given, and there are still doubts on the applicability of this method to other DP steels. Therefore, this identification protocol for different DP steels are investigated in this study, and the results obtained for both DP590 and DP980 show a good agreement with the experimental measurements. 
Additionally, a parametric study has been performed to investigate the robustness of the identification approach. The sensitivity analysis of the identification protocol to each unknown parameter is discussed initially. Subsequently, the influence of the polynomial basis used to construct the surrogate model of fracture strain in local critical elements, is analyzed using computations with bilinear and quadratic bases. Finally, discussion on the identifiability of the protocol to different DP steels is performed by comparing the application of the method to DP590 and DP980 steels.

\section{Experimental work}

In this study, fracture test specimens were extracted from $1.4 \mathrm{~mm}$ thickness cold rolled DP980 steel sheets. And, three groups of experimental fracture tests were performed on a smooth and two notched flat specimens with different notch radius of $R=20 \mathrm{~mm}$ and $R=1 \mathrm{~mm}$. The geometry information of these specimens is shown in Fig. 1. In these tests, the loading axis of each specimen is always located along the rolling direction of the steel. The smooth specimen has a $10 \mathrm{~mm}$ gauge width and a $100 \mathrm{~mm}$ gauge length, while the notched $20 \mathrm{~mm}$ and $1 \mathrm{~mm}$ specimens have the same gauge width but different gauge length of $40 \mathrm{~mm}$ and $20 \mathrm{~mm}$, respectively. Tensile tests have been carried out using Zwick ${ }^{\circledR}$ Z020 universal testing machine with a quasi-static displacement rate of $2 \mathrm{~mm} / \mathrm{min}$ at room temperature. In each test, an electronic extensometer was used to record the displacement. The normalized load-displacement curves are depicted in Fig. 2.

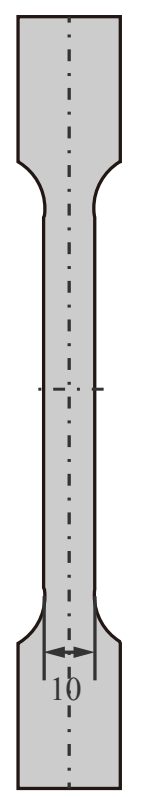

(a)

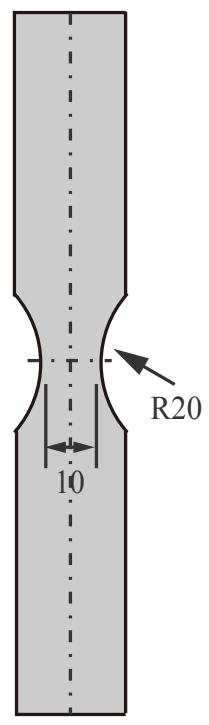

(b)

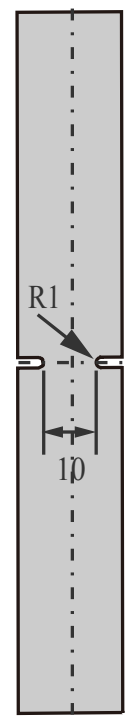

(c)

Figure 1: Geometry information of smooth and notched specimens.

In each test, a maximum force was reached before the fracture. After the force peak, a sudden drop of load took place in the normalized load-displacement curves, which is defined as the onset of final fracture (i.e. the corresponding point indicated by a red star at the end of each curve). And, the fracture onset displacement is represented using the simultaneous relative displacement 

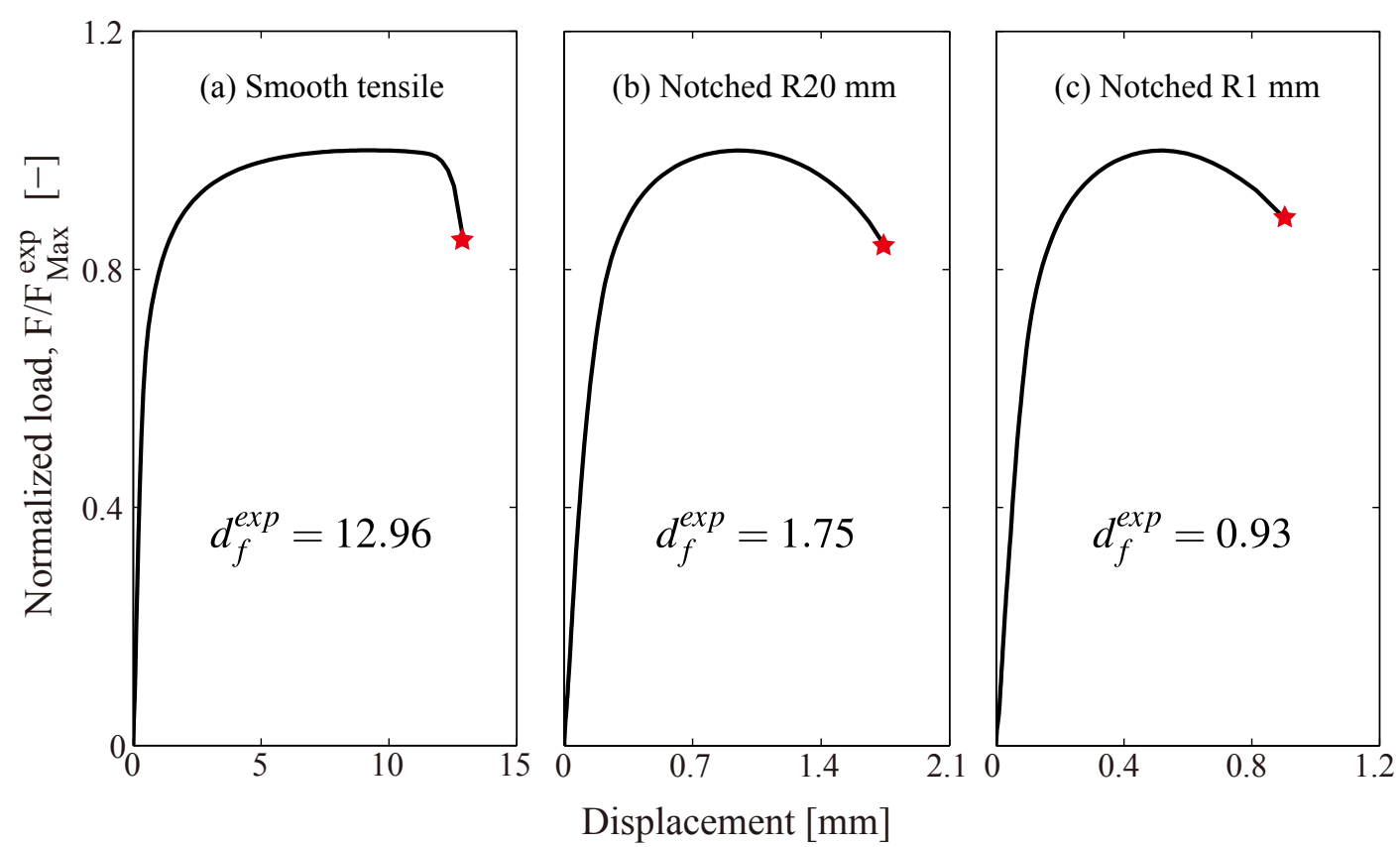

Figure 2: Measured load-displacement curves and average fracture onset displacements for (a) smooth and notched tensile specimens with the notch radius of (b) $20 \mathrm{~mm}$ and (c) $1 \mathrm{~mm}$.

between the test region boundaries. Three specimens of each geometry have been tested to ensure the reproducibility of the results. The variations in the measured fracture displacement are around $2.5 \%$ for smooth specimen, and less than $1.5 \%$ for each notched specimens. The average fracture onset displacement for each specimen is also given in Fig. 2. Moreover, the initial fracture strain $\left(\varepsilon_{f}\right)$ is given by:

$$
\varepsilon_{f}=\ln \left(\frac{A_{0}}{A_{f}}\right)
$$

where $A_{0}$ and $A_{f}$ are initial and fractured cross section areas of a specimen, respectively. Since the cross section after fracture is irregular, this initial fracture strain is considered only as an initial approximation during the computation of exact fracture strain. More detail of the exact fracture strain computation is given in Section 3.2.

\section{Numerical simulation method}

This section focuses on the description of the target fracture criterion and the numerical simulation methods related to the parameter identification procedure.

\subsection{Modified Mohr-Coulomb fracture criterion}

According to the work of Bai and Wierzbicki $[16,17]$, the classical Mohr-Coulomb (MC) criterion has been transformed into the space of stress triaxiality $(\eta)$, normalized Lode angle parameter $(\bar{\theta})$ and equivalent plastic strain to fracture $\left(\bar{\varepsilon}_{f}\right)$ assuming the proportional monotonic loading, in a spherical coordinate system. This fracture criterion is also named as the modified Mohr-Coulomb (MMC) criterion [16], and the explicit expression for the fracture strain is given by: 


$$
\begin{aligned}
\bar{\varepsilon}_{f}(\eta, \bar{\theta})= & \left\{\frac{K}{c_{2}}\left[c_{3}+\left(c_{\theta}-c_{3}\right) \frac{\sqrt{3}}{2-\sqrt{3}}\left(\sec \left(\frac{\pi}{6} \bar{\theta}\right)-1\right)\right]\right. \\
& {\left.\left[\sqrt{\frac{1+c_{1}^{2}}{3}} \cos \left(\frac{\pi}{6} \bar{\theta}\right)+c_{1}\left(\eta+\frac{1}{3} \sin \left(\frac{\pi}{6} \bar{\theta}\right)\right)\right]\right\}^{-\frac{1}{n}} }
\end{aligned}
$$

and

$$
c_{\theta}= \begin{cases}1 & \text { for } \quad \bar{\theta} \geq 0 \\ c_{4} & \text { for } \quad \bar{\theta}<0\end{cases}
$$

where $c_{1}$ is the coefficient of friction and $c_{2}$ is the shear resistance, $c_{3}$ and $c_{\theta}$ describe the dependence of the underlying plasticity model on the third stress invariant. $K$ and $n$ are the fitting parameters obtained using the classical Swift law [23]. The unknown material parameters $\left(c_{1}, c_{2}, c_{3}, c_{4}\right)$ are target parameters which need to be identified based on the fracture experiments. However, due to the application range and nature of the experimental test condition (specimen geometry and loading), the normalized Lode angle parameter $(\bar{\theta})$ is always greater than 0 . Therefore, according to Eq. (3), a simplified three unknown parameter version of the modified Mohr-Coulomb fracture (MMC3) model is obtained by fixing the parameter $c_{\theta}=1$. Hence, the remaining unknown parameters become $\left(c_{1}, c_{2}, c_{3}\right)$.

In Eq. (2), the stress triaxiality is defined by the ratio between the hydrostatic stress $\left(\sigma_{m}\right)$ and von Mises equivalent stress $(\bar{\sigma})$ :

$$
\eta=\frac{\sigma_{m}}{\bar{\sigma}}, \quad \sigma_{m}=\frac{1}{3} \operatorname{tr}(\sigma)
$$

with $-\infty \leq \eta \leq \infty$. The Lode angle parameter is normalized as:

$$
\bar{\theta}=1-\frac{6 \theta}{\pi}, \quad 0 \leq \theta \leq \frac{\pi}{3}
$$

and it lies in the range of $-1 \leq \bar{\theta} \leq 1$. The Lode angle is computed using:

$$
\cos (3 \theta)=\frac{27}{2} \frac{\left(\sigma_{\mathrm{I}}-\sigma_{m}\right)\left(\sigma_{\mathrm{II}}-\sigma_{m}\right)\left(\sigma_{\mathrm{III}}-\sigma_{m}\right)}{\bar{\sigma}^{3}}
$$

where $\sigma_{\mathrm{I}}, \sigma_{\mathrm{II}}$ and $\sigma_{\mathrm{III}}$ are the principal stresses. In the case of proportional loading, the damage evolution indicator $D\left(\bar{\varepsilon}_{p}\right)$ of the fracture is governed by the following an integral formula [16]:

$$
D\left(\bar{\varepsilon}_{p}\right)=\int_{0}^{\bar{\varepsilon}_{p}} \frac{d \bar{\varepsilon}_{p}}{\bar{\varepsilon}_{f}(\eta, \bar{\theta})}
$$

The damage indicator $D\left(\bar{\varepsilon}_{p}\right)$ varies from 0 to 1 , and its initial value for every material element is set as 0 . When $D\left(\bar{\varepsilon}_{p}\right)$ of a material element reaches the threshold value 1 , this element reaches the limit of material ductility and is eliminated from the FE model. Then, the instantaneous displacement that corresponds to the critical element elimination, is referred to as the predicted fracture 
onset displacement, while the exact value is obtained from the experimental measurements. Hence, the identification of the parameters of MMC fracture criterion is performed using an optimization approach to minimize the discrepancy between the predicted and measured results.

\subsection{Bi-level reduced surrogate model using Diffuse Approximation}

As presented in Section 3.1, the three unknown parameter version of MMC fracture criterion that consists of $\left(c_{1}, c_{2}, c_{2}\right)$, has been introduced as the target constitutive model. In order to identify these unknown parameters, a bi-level reduced surrogate model is constructed using Diffuse Approximation.

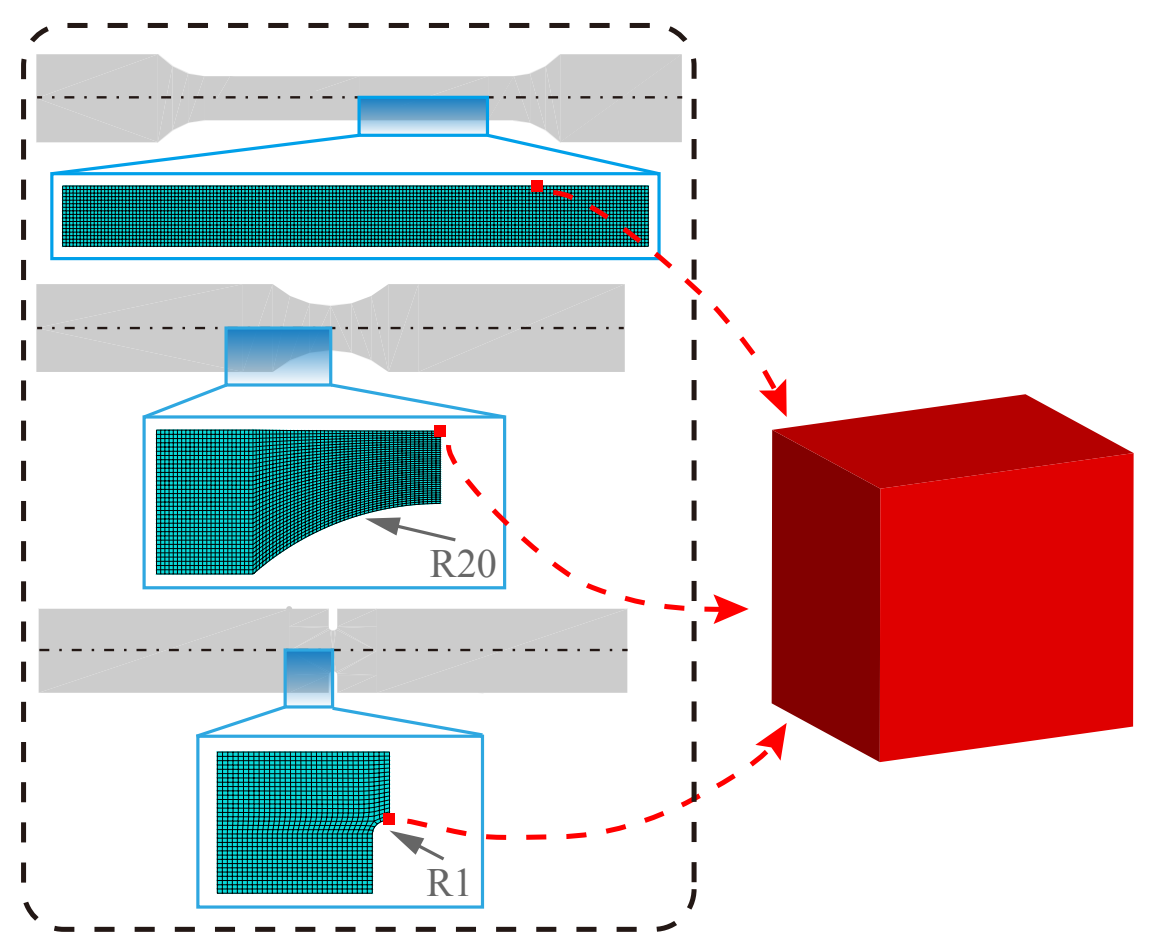

(a) Global FE model

(b) Local critical element

Figure 3: Schematic illustration of the bi-level model: (a) global FE model and (b) local critical element.

To construct the bi-level reduced surrogate model, numerical simulations have been performed for all the specimens described in Section 2. Despite the symmetry of transverse plane in the specimen geometries, the strain localization or fracture initiation does not always occur along the same symmetric plane. Therefore, whole geometry of each specimens is considered in the global FE model to study the fracture behaviors, as shown in Fig. 3a. Each specimen has been meshed with eight-node 3D solid, reduced integration elements (C3D8R in Abaqus/Explicit [24]). Since mesh dependency of MMC3 fracture criterion was discussed in literature [6], a mesh sensitivity study was performed prior to the actual simulation. It was found that, converged results were obtained when the element length was less than or equal to $0.3 \mathrm{~mm}$. Hence, the element length of $0.3 \mathrm{~mm}$ was used in the following numerical simulation to ensure the calculation accuracy and to reduce the computational cost. 
Table 1: Strain hardening parameters of Swift law for DP980 steel.

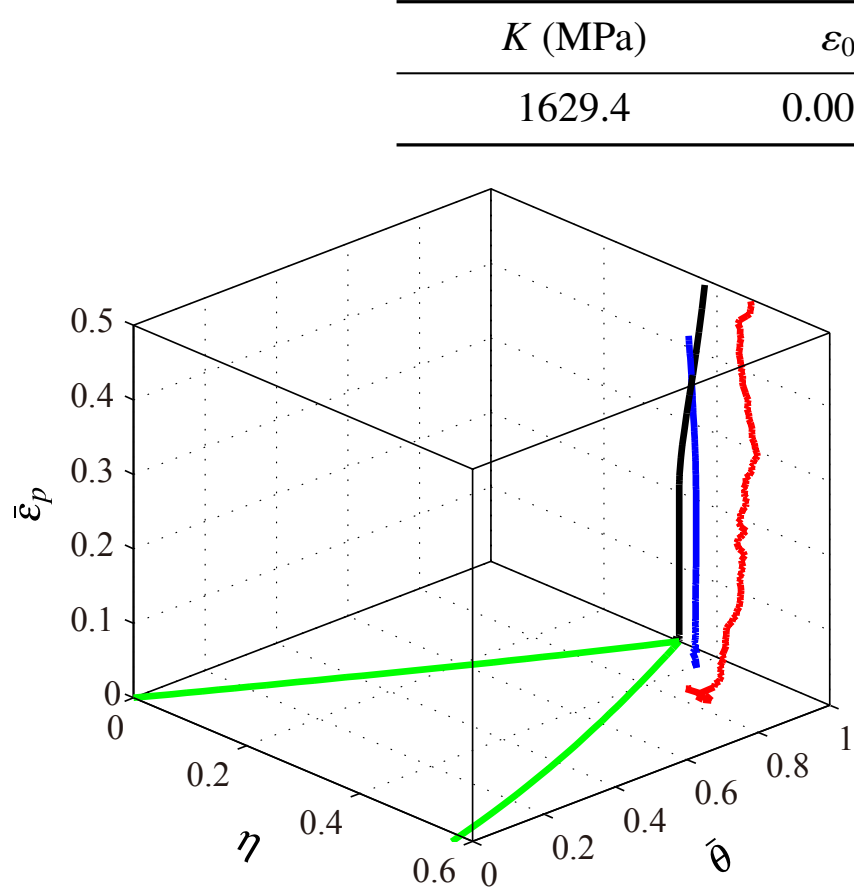

(a)

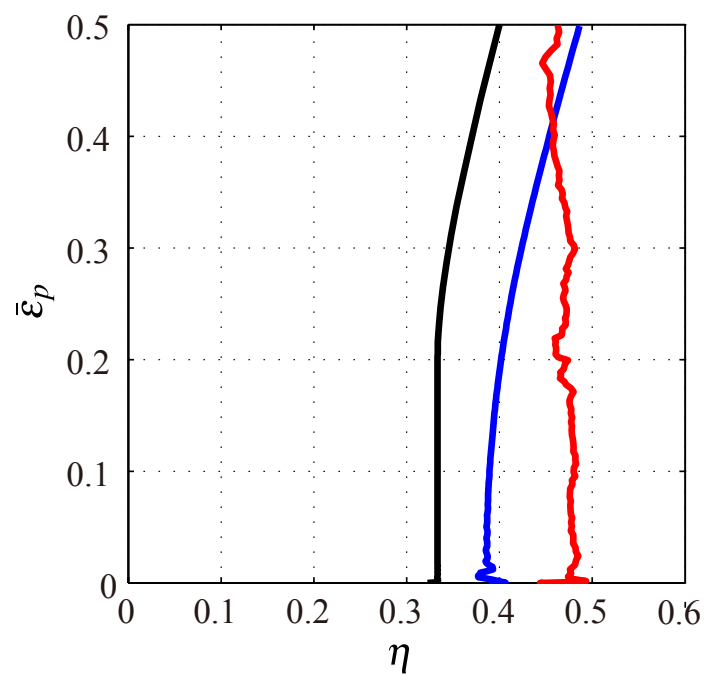

(c)

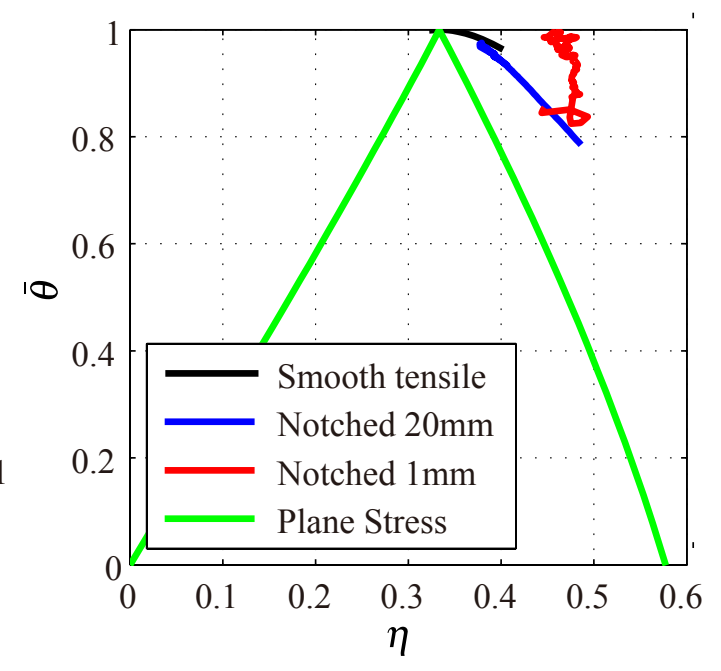

(b)

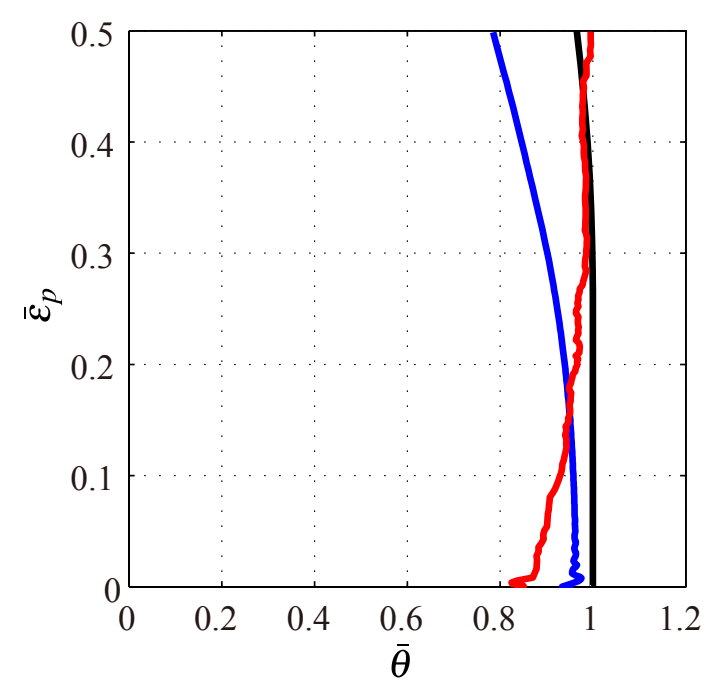

(d)

Figure 4: Plane stress condition and history curves of the stress states and equivalent plastic strain within the initial local critical elements for each specimen in: (a) $\left(\eta, \bar{\theta}, \bar{\varepsilon}_{p}\right)$ space, (b) $(\eta, \bar{\theta})$ plane, (c) $\left(\eta, \bar{\varepsilon}_{p}\right)$ plane and (d) $\left(\bar{\theta}, \bar{\varepsilon}_{p}\right)$ plane.

In order to choose the local critical element in each specimen, an initial FE simulation without fracture criterion has been performed on each specimen combined with the strain hardening parameters given in Table 1. From these FE simulations, according to the strain localization level 
within a particular specimen, the element with highest equivalent plastic strain value is chosen as the local critical element.Moreover, in the following iteration steps, the information of this critical element is verified with the previous iteration step. If it does not remain the same, additional computation (considering the stress state history of a new critical element) is performed within the current iteration step. From numerical simulations, it indicates that, in the smooth and $20 \mathrm{~mm}$ notched tensile specimens, the local critical elements are always located at the center of the longitudinal cross-section, while the third specimen reveals the critical elements along the edge of the open notch, as illustrated in Fig. 3. This dissimilarity is mainly caused by the stress concentration due to the geometric irregularity at the vicinity of the $1 \mathrm{~mm}$ radius notch for the third specimen. The history curves of the stress states (stress triaxiality $\eta$ and normalized Lode angle parameter $\bar{\theta}$ ) and equivalent plastic strain $\bar{\varepsilon}_{p}$ within these local critical elements are plotted in Fig. 4a. And, the values of $\eta, \bar{\theta}$ and $\bar{\varepsilon}_{p}$ are projected on the different individual planes, as illustrated in Figs. $4 \mathrm{~b}$-d.

After that, the MMC3 criterion described in Section 3.1 is programmed in Fortran code as a VUMAT subroutine [24] to predict the fracture onset displacement using numerical simulation. When the critical element is removed from each specimen, the corresponding relative displacement between the test region boundaries is considered as the predicted fracture onset displacement. Moreover, the histories of stress states (i.e., triaxiality and normalized Lode angle parameter) and equivalent plastic strain in each local critical element are recorded with time (see Fig. 4). Based on the paths of stress states, the fracture strain within each local critical element is constructed by the computation of training points $\left(c_{1}, c_{2}, c_{3}\right)$ in DOE. The fracture strain for the whole DOE space corresponding to each iteration is calculated using Diffuse Approximation. During each iteration, a 3D parameter DOE that consists of $H$ points, is designed using the numerical approach described in Section 3.3, expressed by matrix $\mathbf{C}=\left[\mathbf{c}_{1}, \mathbf{c}_{2}, \mathbf{c}_{3}\right]=\left[\mathbf{c}^{1}, \mathbf{c}^{2}, \mathbf{c}^{3}, \ldots, \mathbf{c}^{K}\right]^{T}$.

Let $\boldsymbol{\varepsilon}_{f}(\mathbf{C})$ denote $K \times 3$-dimensional fracture strain computed based on the stress state histories in local critical elements. The surrogate model of the fracture strain estimation is constructed using Diffuse Approximation for the whole DOE space given by:

$$
\tilde{\boldsymbol{\varepsilon}}_{f}(\mathbf{C})=\mathbf{p}(\mathbf{C})^{T} \mathbf{a}(\mathbf{C})
$$

where the plolynomial basis vector $\mathbf{p}$ can be expressed in terms of the unknown parameters for the MMC3 criterion given by:

$$
\mathbf{p}=\left[1, c_{1}, c_{2}, c_{3}, \ldots\right]^{T}
$$

The vector of coefficients $\mathbf{a}=\left[a_{1}, a_{2}, a_{3}, \ldots\right]^{T}$ is the minimizer of the function, defined by:

$$
J(\mathbf{a})=\frac{1}{2} \sum_{k=1}^{K} w\left(\left\|\mathbf{c}-\mathbf{c}^{k}\right\|\right)\left(\mathbf{p}^{T} \mathbf{a}-\boldsymbol{\varepsilon}_{f}\left(\mathbf{c}^{k}\right)\right)^{2}
$$

where $w\left(\left\|\mathbf{c}-\mathbf{c}^{k}\right\|\right)$ is the Gaussian weighting function depending on the Euclidean distance $d$ between the approximated point $\mathbf{c}$ and the neighboring point $\mathbf{c}^{k}$ :

$$
w\left(\left\|\mathbf{c}-\mathbf{c}^{k}\right\|\right)=e^{-\left(\frac{d}{h}\right)^{2}}
$$

in which $h$ is a fixed parameter reflecting the anticipated spacing between the neighboring points [25]. 


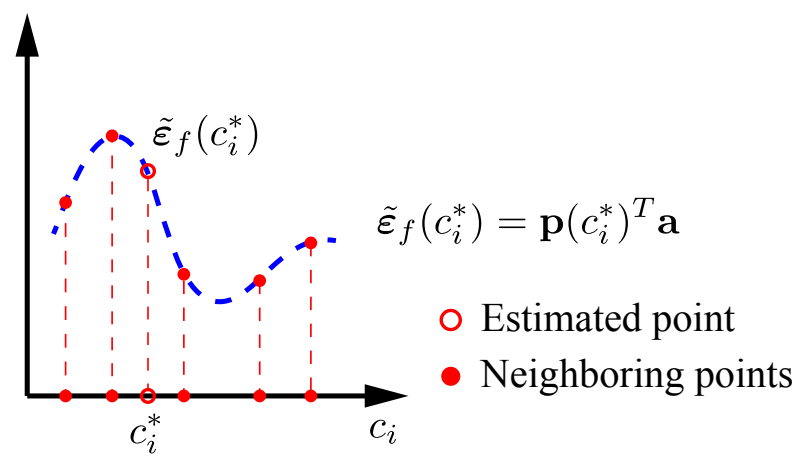

Figure 5: Illustration of the Diffuse Approximation procedure used to construct the surrogate model of fracture strain prediction.

It is used to smooth the small fluctuation in the data.

Fig. 5 illustrates the estimation procedure, for a given fracture strain value of DOE training point. The corresponding approximated values for the whole design space are locally interpolated using Diffuse Approximation. Therefore, we construct the surrogate model including the LSE. between approximated and measured fracture strain for each specimen, given by:

$$
\operatorname{Argmin} L S E .=\frac{1}{m} \sum_{i=1}^{m}\left(\tilde{\varepsilon}_{f i}(\mathbf{c})-\varepsilon_{f i}^{e x p}\right)^{2}
$$

where $\tilde{\varepsilon}_{f i}(\mathbf{c})$ is the predicted fracture strain at the point $\mathbf{c}, \varepsilon_{f i}^{\exp }$ is the measured fracture strain, and $m$ is the number of various specimen geometries. The obtained parameters are then introduced within the global FE simulation to compute the corresponding LSE. of the fracture onset displacement:

$$
\operatorname{Argmin} L S E .=\frac{1}{m} \sum_{i=1}^{m}\left(d_{f i}-d_{f i}^{e x p}\right)^{2}
$$

where $d_{f i}$ and $d_{f i}^{e x p}$ are predicted and measured fracture onset displacements, respectively.

During the parameter identification procedure, a quadratic polynomial basis (2nd-order), which includes 10 terms, is used to construct the surrogate model given in Eq. (8). Moreover, 11 numerical training points are selected from the successive DOE space to compute the corresponding fracture strain using the histories of stress states and Eq. (2). By minimizing the LSE. in Eq. (12), the identified parameters are determined in each iteration. Then, the identified material parameters are introduced in the global FE fracture simulations. To launch the identification procedure, the initial fracture strains computed by Eq. (1) are used as the first approximation of the fracture strains. However, due to the absence of high-precision measurement technique, such as the digital image correlation (DIC) technique, the simultaneous fracture strains are not captured effectively. Therefore, an adaptive correlation between the fracture onset displacement and strain is introduced to compute the exact fracture strain values. In each iteration, an adaptive deviation of fracture onset displacement is computed by:

$$
\Delta d_{f i}=d_{f i}-d_{f i}^{e x p}
$$




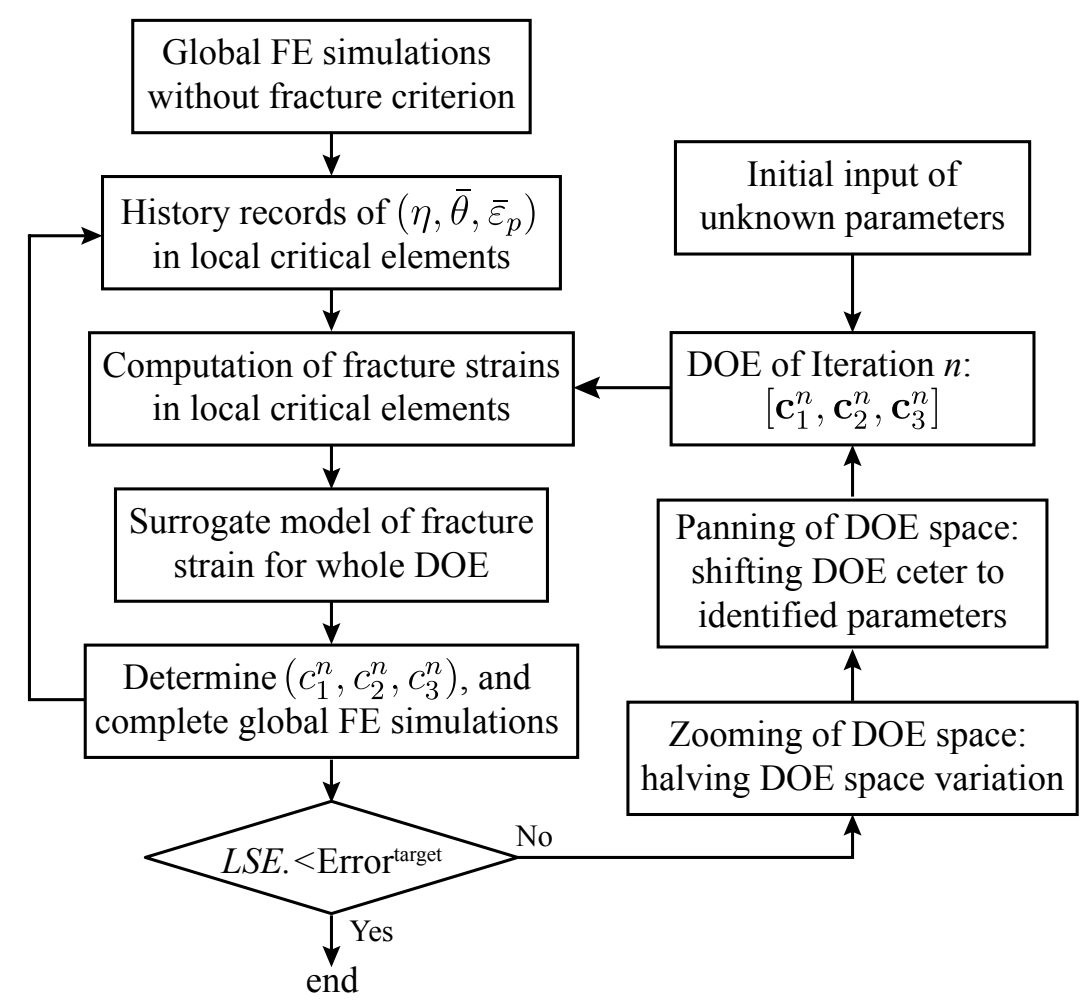

Figure 6: Flow chart of the parameter identification process using the proposed bi-level reduced surrogate model.

Then, the new fracture strain is obtained using the adaptive deviation:

$$
\left(\varepsilon_{f i}^{\text {exp }}\right)_{\text {new }}=\left(\varepsilon_{f i}^{\text {exp }}\right)_{\text {old }}+\ln \left(1+\frac{\Delta d_{f i}}{L_{0 i}}\right)
$$

where $d_{f i}$ and $d_{f i}^{e x p}$ are the fracture onset displacements obtained from the global FE simulation and experimental measurements, respectively. $L_{0 i}$ is the initial effective length in the test region of each specimen. The adaptive correlation between the displacement and strain at the fracture onset ensures that the fracture strain target varies around the exact value with decreasing error that eventually becomes negligible. Finally, the unknown parameters of MMC3 fracture criterion are identified using the convergence condition given in Eq. (13). Therefore, the proposed scheme provides high accuracy of the ductile fracture calibration procedure.

Additionally, the DOE of next iteration is designed by shifting the center to the identified parameters and halving the DOE variation. Converged material parameters are identified when the variation of DOE space and $L S E$. of the fracture onset displacement (in Eq. (13)) reach sufficiently acceptable ranges. Further details of the successive DOE approach are given in the following section. The flow chart of the whole identification strategy is illustrated in Fig. 6. The proposed bilevel modeling reduction strategy guarantees not only the accuracy of identification process while using a limited number of experimental tests, but also significantly reduces the computational time. 


\subsection{Successive DOE}

Successive DOE approach is combined with the bi-level reduced surrogate model when identifying the unknown parameters using an iterative algorithm. Fig. 7 shows the schematic illustration of the successive DOE. In each iteration step, a set of initial estimation (blue dots in Fig. 7) is considered as the center of DOE design space. Moreover, a Latin Hypercube Sampling (LHS) approach is employed to design 11 numerical training points (black dots in Fig. 7) within a sufficiently large design space. By using the bi-level reduced surrogate model and the objective function in Eq. (12), optimum point (red dots in Fig. 7) is identified for each iteration.

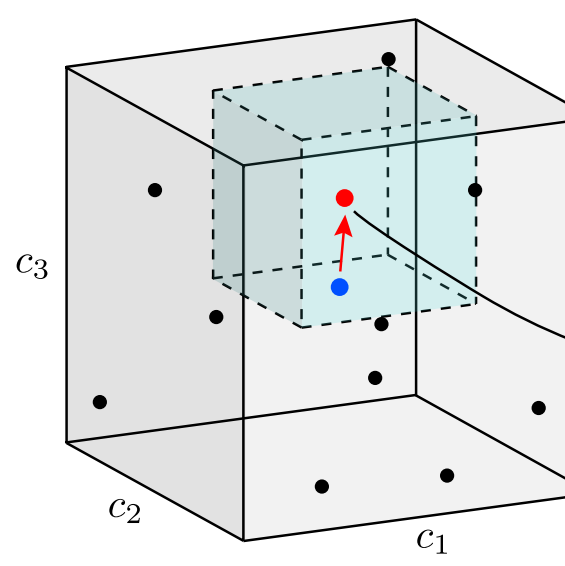

Iteration. 1

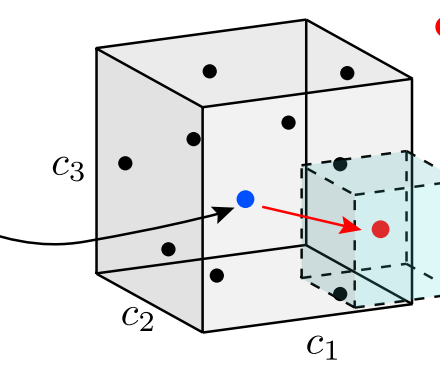

Iteration. 2
- Initial estimation

- Numerical training point

- Identified point

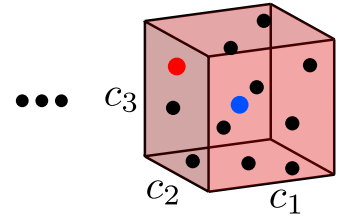

Iteration. i

Figure 7: Schematic illustration of the successive DOE design.

Then, two operations called as panning and zooing algorithms [? ], are used to implement the transformation of DOE space. Firstly, the center of DOE design space is transfered from the initial estimated point to the identified point (indicated by red arrows in Fig. 7). Secondly, the variation of the new DOE space is halved to narrow down the searching range (azure color cubes with dushed line frame in Fig. 7). Finally, when the computation enter the next iteration, new group of training points are reselected within a new DOE space. During the iterative identification procedure, the initial and identified points may be overlapped or located nearby. DOE space design and transformation is repeated until a convergence is reached.

\section{Results and discussion}

\subsection{Parameter identification of MMC3 criterion for DP980 steel}

The parameters $\left(c_{1}, c_{2}, c_{3}\right)$ of MMC3 fracuture criterion are identified using the numerical method presented in Section 3 for DP980 steel. Initial values of input fracture onset displacement and fracture strain are extracted from the experimental measurements described in Section 2. Moreover, the initial estimation of the unknown parameters is set as $(0.2,800 \mathrm{MPa}, 1.03)$ based on the experience of numerical experiments. Besides, the DOE space for the first iteration is located in the domain: $c_{1} \in[0,0.4], c_{2} \in[600,1000]$ (unit: $\mathrm{MPa}$ ) and $c_{3} \in[0.8,1.5]$.

Fig. 8 shows the LSE. between the measured and predicted fracture onset displacements for each iteration. It clearly indicates that, the $L S E$. fiercely decreases to 0.226 until Iteration\#4. 


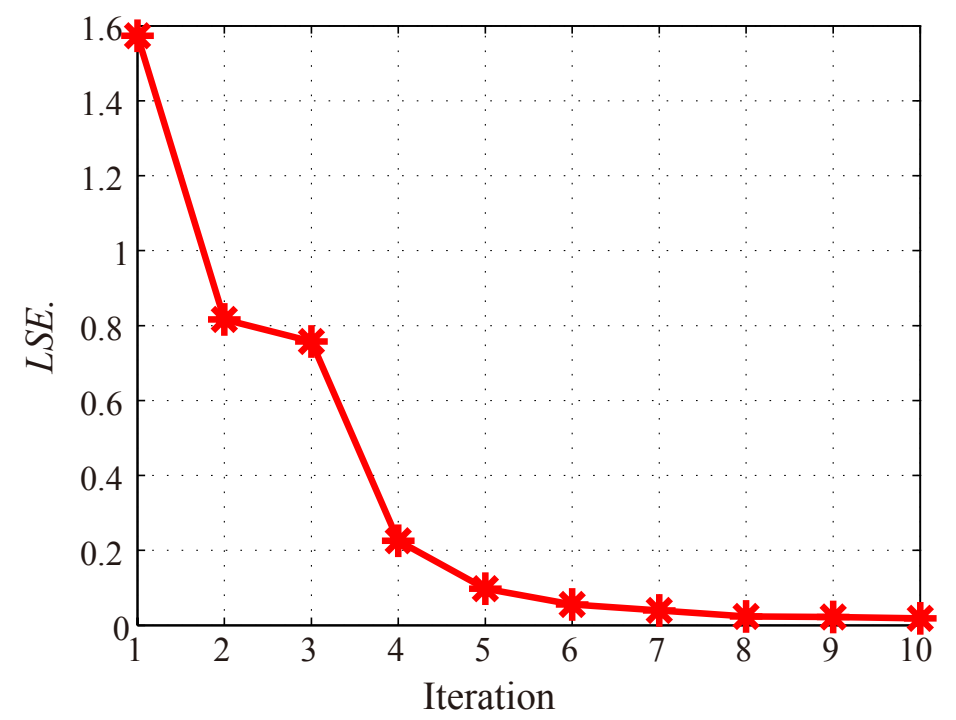

Figure 8: Progressive trend of the LSE. between the measured and predicted fracture onset displacements with the identified material parameters for each iteration.
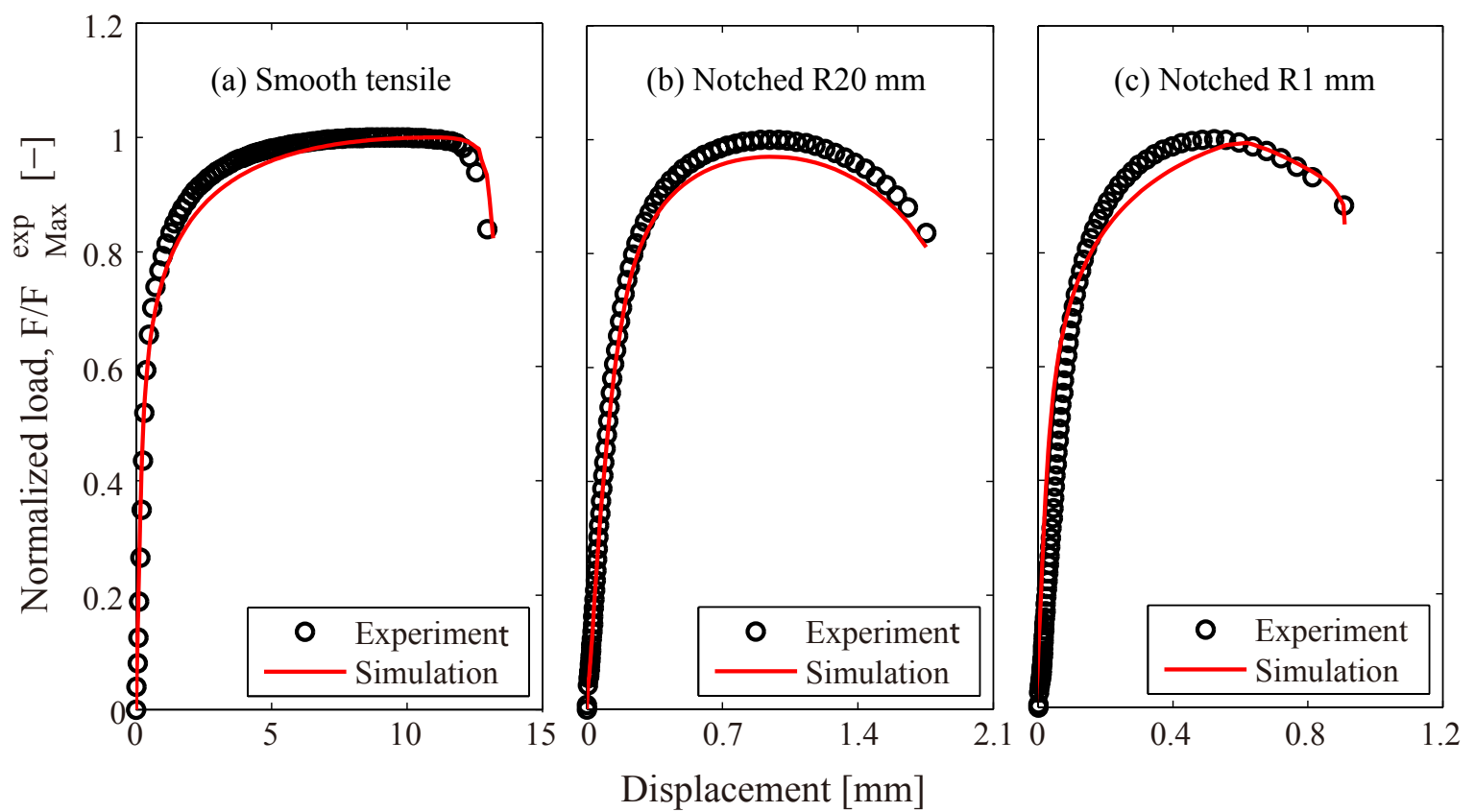

Figure 9: Comparisons between the measured and predicted load-displacement curves for (a) smooth and notched tensile specimens with the notch radius of (b) $20 \mathrm{~mm}$ and (c) $1 \mathrm{~mm}$. The identified MMC3 parameters are used in the numerical simulation.

Especially, during Iteration\#2 and 4, the value of LSE. is reduced significantly. After that, the curve gradually decreases with subsequent iterations, and the $L S E$. stabilizes with 0.018 at Iteration\#10, which is smaller than the target $L S E$. value (0.020). Therefore, the material parameters $\left(c_{1}, c_{2}, c_{3}\right)$ of MMC3 criterion are finally identified as $(0.178,896.734 \mathrm{MPa}, 1.043)$ for DP980 steel.

By introducing these identified parameters into the numerical simulation, the load-displacement 
curve is computed using the VUMAT subroutine for each specimen. Fig. 9 shows comparisons between the measured and predicted load-displacement curves. It is found that, the predicted values of fracture onset displacement concur with the experimental measurements. It confirms the robustness of the parameter identification approach using the proposed bi-level reduced surrogate model.

\subsection{Sensitivity to different parameters}

In the previous section, the unknown parameters $\left(c_{1}, c_{2}, c_{3}\right)$ of MMC3 fracture criterion have been identified for DP980 steel using the bi-level reduced surrogate model. Due to the nonlinearity of MMC3 fracture criterion, the sensitivity of the identification algorithm to different parameters is not the same. Therefore, this section focuses on the sensitivity analysis, which is performed to further understand the identifiability of ductile fracture criterion using bi-level reduced surrogate model.

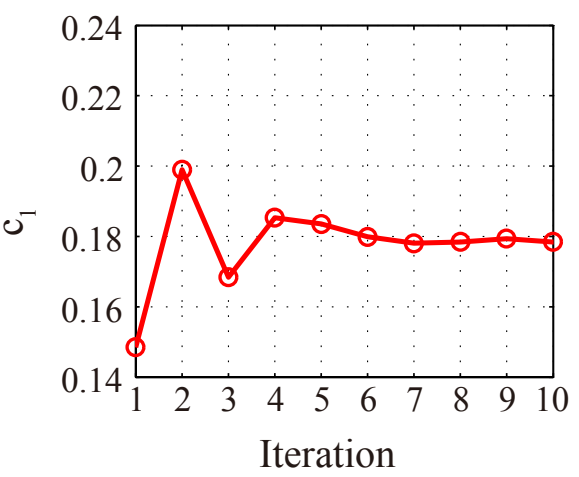

(a)

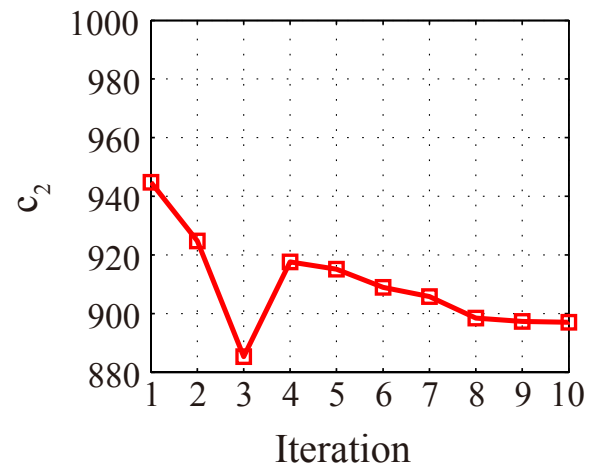

(b)

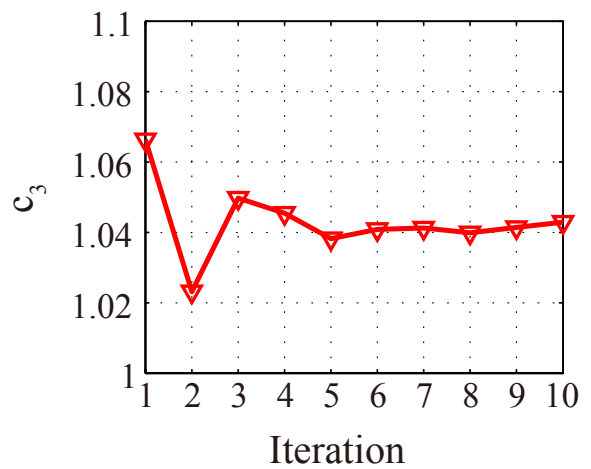

(c)

Figure 10: Convergence curves obtained for parameters (a) $c_{1}$, (b) $c_{2}$ (unit: MPa) and (c) $c_{3}$.

Convergence curves for $c_{1}, c_{2}$ and $c_{3}$ against the iteration step are depicted in Fig. 10a, b and c, respectively. Moreover, to capture the sensitivity of the proposed identification algorithm for each parameter, relative error between the current and final identified parameters of each iteration step is expressed as:

$$
\varepsilon\left(c_{i}\right)=\frac{\left|c_{i}-c_{i}^{*}\right|}{c_{i}^{*}}
$$


Table 2: Iteration results for different MMC3 parameters.

\begin{tabular}{ccccccc}
\hline Iteration & $c_{1}$ & $c_{2}(\mathrm{MPa})$ & $c_{3}$ & $\varepsilon\left(c_{1}\right)$ & $\varepsilon\left(c_{2}\right)$ & $\varepsilon\left(c_{3}\right)$ \\
\hline 1 & 0.148 & 944.737 & 1.066 & $16.77 \%$ & $5.35 \%$ & $2.24 \%$ \\
2 & 0.199 & 924.726 & 1.023 & $11.56 \%$ & $3.12 \%$ & $1.90 \%$ \\
3 & 0.168 & 885.288 & 1.050 & $5.55 \%$ & $1.28 \%$ & $0.65 \%$ \\
4 & 0.185 & 917.632 & 1.045 & $3.94 \%$ & $2.33 \%$ & $0.23 \%$ \\
5 & 0.184 & 915.133 & 1.038 & $2.93 \%$ & $2.05 \%$ & $0.46 \%$ \\
6 & 0.180 & 908.882 & 1.041 & $0.90 \%$ & $1.35 \%$ & $0.21 \%$ \\
7 & 0.178 & 905.757 & 1.041 & $0.11 \%$ & $1.01 \%$ & $0.17 \%$ \\
8 & 0.178 & 898.420 & 1.040 & $0.06 \%$ & $0.19 \%$ & $0.30 \%$ \\
9 & 0.179 & 897.341 & 1.042 & $0.56 \%$ & $0.07 \%$ & $0.16 \%$ \\
10 & 0.178 & 896.734 & 1.043 & $0.0 \%$ & $0.0 \%$ & $0.0 \%$ \\
\hline
\end{tabular}

where $c_{i}^{*}$ and $c_{i}$ denote the identified parameters of current and final iteration steps, respectively. Hence, further details of the iteration results are given in Table 2. For parameter $c_{1}$, the optimal value after Iteration\#1 is identified as 0.148 , and the initial relative error is $16.77 \%$. The convergence curve stabilizes after Iteration\#6, which corresponds to the relative error $\varepsilon\left(c_{1}\right)$ of $0.90 \%$. Then, the convergence curve shows a negligible variation around the final identified value $(0.178)$ until the LSE. of fracture onset displacements minimizes below the pre-defined threshold. Meanwhile, the convergence curve of parameter $c_{2}$ continues to decrease until Iteration\#6. After that, the curve reaches the convergent value at Iteration\#8, with the relative error of less than $0.19 \%$ (Fig. 10b). While, the convergence curve of parameter $c_{3}$ reaches to 1.050 at Iteration\#3, with the relative error of $0.65 \%$ (Fig. 10c).

By comparing the convergence curve and relative error of each parameter, it is found that, although the initial value of $\varepsilon\left(c_{1}\right)$ is significantly larger than that of two other cases, it requires five iterations to fiercely reach the relative error below $1.0 \%$. On the contrary, the initial value of $\varepsilon\left(c_{2}\right)$ is much smaller, but it requires seven iterations to become less than $1.0 \%$. In the case of parameter $c_{3}$, the optimal value at Iteration\#1 is closer to the final identified result (the relative error is $2.24 \%$ ) than the other two parameters. It takes two iterations to minimize the relative error $\varepsilon\left(c_{3}\right)$ below $1.0 \%$. Hence, we can conclude that the identification algorithm is more sensitive to parameter $c_{1}$ than to the other parameters. In a given case where the initial relative error of each parameter begins with the same value, the convergence curve of parameter $c_{2}$ will take more iteration steps to obtain the final identified value. In other words, the total iteration number of the whole identification process mainly depends on the initial estimation of parameter $c_{2}$.

\subsection{Effect of polynomial basis}

In this study, the surrogate model of fracture strain in local critical elements is approximated based on a set of training points in the vicinities of current estimation point. Since it allows for a gradient and Hessian evaluation, a quadratic polynomial basis is employed throughout the identification processes. However, the number of coefficients of quadratic basis increases significantly with the number of parameters. Hence, it is necessary to investigate a lower order bilinear basis 
to reduce the number of training points per iteration. Especially, the lower order polynomial basis behaves efficiently and prominently when more parameters are involved in the identification process. For example, for a case where 6 parameters are involved, the quantities of training points per iteration required for bilinear and quadratic bases are 22 and 28, respectively.

Table 3: Identified results derived by applying different polynomial bases.

\begin{tabular}{ccccccc}
\hline Polynomial basis & $c_{1}$ & $c_{2}(\mathrm{MPa})$ & $c_{3}$ & Iterations & Training points & Computations \\
\hline Bilinear & 0.176 & 897.017 & 1.041 & 16 & 8 & 128 \\
Quadratic & 0.178 & 896.734 & 1.043 & 10 & 11 & 110 \\
\hline
\end{tabular}

To study the effect of polynomial basis, a comparison between bilinear and quadratic bases is performed by setting the same initial estimation for the unknown parameters $\left(c_{1}, c_{2}, c_{3}\right)$ identification. The identified results derived by applying different polynomial bases are given in Table 3 . Almost similar parameters are identified by adopting both bilinear and quadratic polynomial bases. It is also found that, the number of training points per iteration for bilinear basis are less than that for quadratic basis ( 8 compared with 11), but the total number of computations is more (128 compared with 110). Furthermore, each iteration step includes the calculations not only for the local critical elements but also for the global FE simulations, and the latter one costs significantly extra times of local computational efforts than the former one. Commonly, the computational time associated with the global FE simulations is approximately 2.5 hours for each iteration on a work station with 2 Intel ${ }^{\circledR}$ Xeon ${ }^{\circledR}$ E5-2667 v4 processors $(2 \times 8$ cores, $3.2 \mathrm{GHz})$, while the local calculation with 11 training points only requires around 35 seconds. However, when using the quadratic basis, it significantly reduces the total number of iteration steps, thus it reduces the required number of global FE simulations. Therefore, the quadratic basis is relatively suitable for this identification strategy.

\subsection{Applications to different DP steels}

In our previous work [6], the material parameters of MMC3 fracture criterion had been identified for DP590 steel using the bi-level reduced surrogate model. The identification process was carried out with the initial estimation of the three free parameters $(0.2,800 \mathrm{MPa}, 1.03)$, and continued for 11 iteration steps to obtain the converged MMC3 parameters of $(0.117,567.784 \mathrm{MPa}$, 0.982). The LSE. between the measured and predicted fracture onset displacements stabilized at around 0.02. Furthermore, parameter identification had been performed with different initial estimation, and the similar results were obtained. This also indicates the robustness of this identification protocol to identify the material parameters of MMC3 fracture criterion for different materials.

To further investigate the identifiability of this protocol to different DP steels, numerical results obtained for DP590 steel have been compared with those for DP980 steel. Fig. 11 shows the fracture onset displacement errors between the measured and predicted results for these two steels. In Fig. 11, only slight fracture onset displacement errors are observed for each DP steel, it means that the predicted results corroborate well with the experimental measurements. In the case of DP590 steel, all the measured fracture onset displacements are greater than the predicted ones, 


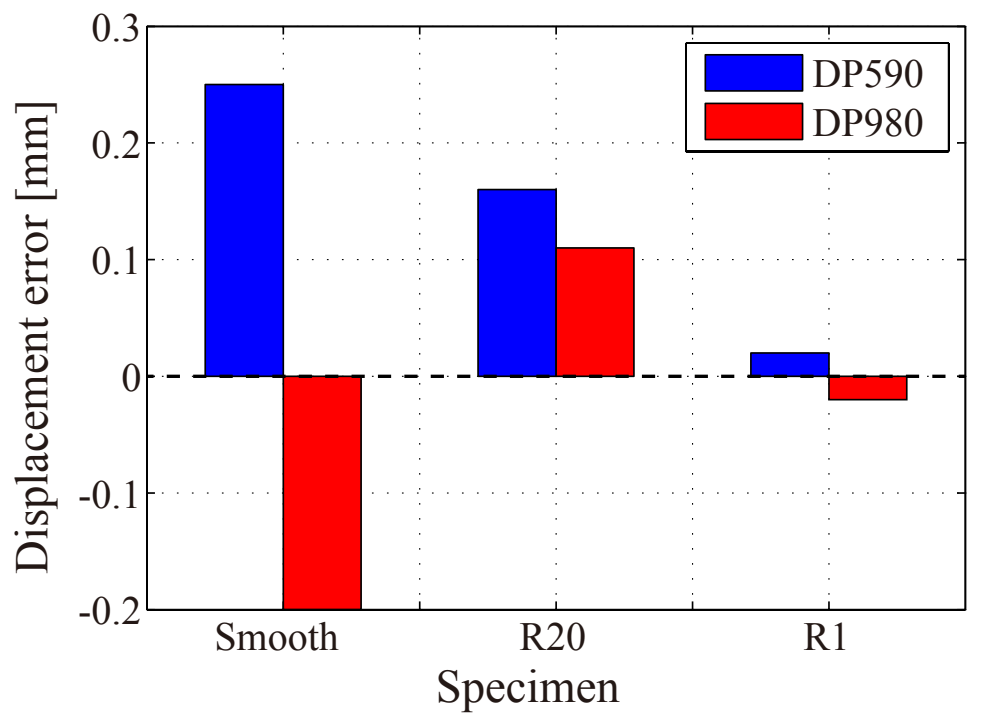

Figure 11: Fracture onset displacement error between the measured and predicted results for DP590 and DP980 steels. The identified MMC3 parameters are used in the numerical simulation.

which leads all the corresponding errors to be positive. However, for DP980 steel, the positive fracture displacement error appears only in the fracture test of notched specimen with the notch radius of $20 \mathrm{~mm}$. By comparing the absolute values of these errors, the maximum error appears in the fracture tests of smooth specimen in both cases, and their real values are $0.25 \mathrm{~mm}$ and $0.20 \mathrm{~mm}$ for DP590 and DP980 steels, respectively. The other two displacement errors are 0.16 $\mathrm{mm}$ and $0.02 \mathrm{~mm}$ for DP590 steel, while they are $0.11 \mathrm{~mm}$ and $-0.02 \mathrm{~mm}$ for DP 980 steel. The reasonable explanation is that, the final displacement after fracture of smooth specimen is essentially longer than those of the notched specimens. Hence, the smooth specimen possesses the maximum error. Based on the comparison, it can be concluded that, the identification protocol is effective to calibrate the MMC3 parameters and to capture the ductile fracture behaviors for different DP steels.

\section{Summary and outlook}

In this study, the identifiability of modified Mohr-Coulomb (MMC) fracture criterion with a limited number of fracture tests was investigated using a bi-level reduced surrogate model for DP steels. By employing the successive DOE algorithm, satisfactory results have been obtained via the identification process. The main contributions are listed as follows:

- The bi-level reduced surrogate model provides a novel orientation in capturing the ductile fracture behaviors of metallic materials. By combining the local critical element and global FE models, unknown parameters of a given fracture model were identified using successive DOE. The least square error of fracture onset displacement between the experimental and predicted results was controlled at a considerable low level of less than $2 \%$.

- Sensitivity analysis of the identification approach to each parameter was investigated by 
comparing the covergence curve and relative error of each parameter at every iteration. It is observed that, the proposed protocol is more sensitive to $c_{1}$ than $c_{2}$ and $c_{3}$.

- Effect of the polynomial basis on the construction of the surrogate model was studied by employing bilinear and quadratic bases using the same initial estimation of unknown parameters. The application of bilinear basis reduces the number of training points per iteration, but required more iteration steps to obtain the converged results. Consequently, the bilinear basis requires more total computational time to search for the identification parameters.

- Parameter identification was performed on DP590 and DP980 steels, minor errors of fracture onset displacement were found by comparing the predicted and measured results for each fracture test. It confirms that, the identification protocol suits for different DP steels to identify the MMC3 parameters.

The proposed protocol thus allows to calibrate parameters of MMC3 fracture criterion with a limited quantity of experimental tests. Using this model, further improvements and investigations are possible by considering the inaccuracy of experimental measurements, the intrinsic dimensionality of the local fracture strain surrogate models, the possibility of the identification protocol for other elastoplastic [11, 12] or fracture constitutive models of various metallic and composite materials $[26,27]$. Moreover, this bi-level reduced surrogate modeling approach is likely to be extended to search the effective solution for structural optimization problems [28-31].

\section{Acknowledgements}

Authors acknowledge the financial support from ESI-Group and Natural Science Foundation of China (NSFC, No.U1833116). This study is also supported by China Postdoctoral Science Foundation (No.2018M642775) and Henan Province Science and Technology Key Project (No.182102210186).

\section{References}

[1] X. Sun, E. V. Stephens, M. A. Khaleel, Effects of fusion zone size and failure mode on peak load and energy absorption of advanced high strength steel spot welds under lap shear loading conditions, Engineering Failure Analysis 15 (4) (2008) 356-367.

[2] V. Uthaisangsuk, U. Prahl, W. Bleck, Modelling of damage and failure in multiphase high strength DP and TRIP steels, Engineering Fracture Mechanics 78 (3) (2011) 469-486.

[3] S. Dancette, D. Fabrègue, V. Massardier, J. Merlin, T. Dupuy, M. Bouzekri, Investigation of the tensile shear fracture of advanced high strength steel spot welds, Engineering Failure Analysis 25 (2012) 112-122.

[4] J. Wang, S. Zhou, Z. Zhang, D. Yurchenko, High-performance piezoelectric wind energy harvester with y-shaped attachments, Energy Conversion and Management 181 (2019) 645-652.

[5] Y. Hou, T. Sapanathan, A. Dumon, P. Culière, M. Rachik, A novel artificial dual-phase microstructure generator based on topology optimization, Computational Materials Science 123 (2016) 188-200.

[6] Y. Hou, T. Sapanathan, A. Dumon, P. Culière, M. Rachik, A novel development of bi-level reduced surrogate model to predict ductile fracture behaviors, Engineering Fracture Mechanics 188 (2018) 232-249.

[7] Y. Hou, S. Cai, T. Sapanathan, A. Dumon, M. Rachik, Micromechanical modeling of the effect of phase distribution topology on the plastic behavior of dual-phase steels, Computational Materials Science 158 (2019) 243-254. 
[8] Q. Liu, Q. Zhou, J. Venezuela, M. Zhang, A. Atrens, Evaluation of the influence of hydrogen on some commercial DP, Q\&P and TWIP advanced high-strength steels during automobile service, Engineering Failure Analysis 94 (2018) 249-273.

[9] Y. Bao, T. Wierzbicki, A comparative study on various ductile crack formation criteria, Journal of engineering materials and technology 126 (3) (2004) 314-324.

[10] T. Borsato, P. Ferro, F. Berto, C. Carollo, Mechanical and fatigue properties of pearlitic ductile iron castings characterized by long solidification times, Engineering Failure Analysis 79 (2017) 902-912.

[11] L. Meng, P. Breitkopf, G. Le Quilliec, An insight into the identifiability of material properties by instrumented indentation test using manifold approach based on $\mathrm{Ph}$ curve and imprint shape, International Journal of Solids and Structures 106 (2017) 13-26.

[12] L. Meng, P. Breitkopf, B. Raghavan, G. Mauvoisin, O. Bartier, X. Hernot, On the study of mystical materials identified by indentation on power law and Voce hardening solids, International Journal of Material Forming (2018) 1-16.

[13] L. Meng, W. Zhang, D. Quan, G. Shi, L. Tang, Y. Hou, P. Breitkopf, J. Zhu, T. Gao, From topology optimization design to additive manufacturing: Today's success and Tomorrow's roadmap, Archives of Computational Methods in Engineering (2019) 1-25.

[14] X. Yang, Y. Liu, X. Fang, C. Mi, Estimation of low failure probability based on active learning kriging model with a concentric ring approaching strategy, Structural and Multidisciplinary Optimization 58 (3) (2018) 11751186.

[15] X. Yang, C. Mi, D. Deng, Y. Liu, A system reliability analysis method combining active learning kriging model with adaptive size of candidate points, Structural and Multidisciplinary Optimization (2019) 1-14.

[16] Y. Bai, T. Wierzbicki, A new model of metal plasticity and fracture with pressure and Lode dependence, International journal of plasticity 24 (6) (2008) 1071-1096.

[17] Y. Bai, T. Wierzbicki, Application of extended Mohr-Coulomb criterion to ductile fracture, International Journal of Fracture 161 (1) (2010) 1.

[18] M. Dunand, D. Mohr, On the predictive capabilities of the shear modified Gurson and the modified MohrCoulomb fracture models over a wide range of stress triaxialities and Lode angles, Journal of the Mechanics and Physics of Solids 59 (7) (2011) 1374-1394.

[19] S. Cicero, T. García, V. Madrazo, I. Carrascal, E. Ruiz, Analysis of notch effect in load bearing capacity, apparent fracture toughness and fracture micromechanisms of ferritic-pearlitic steels, Engineering Failure Analysis 44 (2014) 250-271.

[20] N. Habibi, A. Ramazani, V. Sundararaghavan, U. Prahl, Failure predictions of DP600 steel sheets using various uncoupled fracture criteria, Engineering Fracture Mechanics 190 (2018) 367-381.

[21] L. Xia, B. Raghavan, P. Breitkopf, W. Zhang, Numerical material representation using proper orthogonal decomposition and diffuse approximation, Applied Mathematics and Computation 224 (2013) 450-462.

[22] L. Xia, P. Breitkopf, Multiscale structural topology optimization with an approximate constitutive model for local material microstructure, Computer Methods in Applied Mechanics and Engineering 286 (2015) 147-167.

[23] H. Swift, Plastic instability under plane stress, Journal of the Mechanics and Physics of Solids 1 (1) (1952) 1-18.

[24] Hibbitt, Karlsson, Sorensen, ABAQUS/Explicit: user's manual, Vol. 1, Hibbitt, Karlsson and Sorenson Incorporated, 2001.

[25] P. Breitkopf, H. Naceur, A. Rassineux, P. Villon, Moving least squares response surface approximation: Formulation and metal forming applications, Computers \& Structures 83 (17-18) (2005) 1411-1428.

[26] Y. Xu, T. You, C. Du, An integrated micromechanical model and bp neural network for predicting elastic modulus of 3-d multi-phase and multi-layer braided composite, Composite Structures 122 (2015) 308-315.

[27] Y. Xu, P. Zhang, W. Zhang, Two-scale micromechanical modeling of the time dependent relaxation modulus of plain weave polymer matrix composites, Composite Structures 123 (2015) 35-44.

[28] Y. Hou, Y. Tie, C. Li, T. Sapanathan, M. Rachik, Low-velocity impact behaviors of repaired cfrp laminates: Effect of impact location and external patch configurations, Composites Part B: Engineering 163 (2019) 669_ 680 .

[29] Y. Wang, Z.-P. Wang, Z. Xia, L. H. Poh, Structural design optimization using isogeometric analysis: a Comprehensive Review, CMES-Computer Modeling in Engineering and Science 117 (3) (2018) 455-507. 
[30] L. Xia, L. Zhang, Q. Xia, T. Shi, Stress-based topology optimization using bi-directional evolutionary structural optimization method, Computer Methods in Applied Mechanics and Engineering 333 (2018) 356-370.

[31] Z. Wu, L. Xia, S. Wang, T. Shi, Topology optimization of hierarchical lattice structures with substructuring, Computer Methods in Applied Mechanics and Engineering 345 (2019) 602-617. 